\title{
铁催化硅氢加成反应研究进展
}

\author{
徐大鹏肖文军*彭家建*厉嘉云白赢
}

(杭州师范大学有机硅化学及材料技术教育部重点实验室 杭州 311121)

\begin{abstract}
摘要 铁催化剂应用于催化硅氢加成反应，具有原料丰富度高、低成本、低毒性等优点，在近几年得到迅速发展. 总结 了铁催化剂在碳一碳双键、碳一氧双键及其他杂原子不饱和双键硅氢加成反应中的应用, 并展望了铁催化剂在有机硅化 学领域的应用前景.
\end{abstract}

关键词 铁配合物; 硅氢加成; 碳一碳双键; 杂原子双键; 进展

\section{Progress in Hydrosilylation Catalyzed with Iron Catalyst}

\author{
Xu, Dapeng Xiao, Wenjun* Peng, Jiajian* Li, Jiayun Bai, Ying \\ (Key Laboratory of Organosilicon Chemistry and Material Technology, Ministry of Education, \\ Hangzhou Normal University, Hangzhou 311121)
}

\begin{abstract}
Iron catalysts gained great development in hydrosilylation of various types of unsaturated double bond compounds due to their advantages of high raw material abundance, low cost, low toxicity, etc. The applications of iron catalysts are summarized in the hydrosilylation of carbon-carbon double bond, carbon-oxygen double bond and other heteroatomic double bond. The application prospect of iron catalysts in the field of organic silicon chemistry is looked forward.

Keywords iron complex; hydrosilylation; carbon-carbon double bond; heteroatomic double bond; progress
\end{abstract}

硅氢加成反应是生成 $\mathrm{Si}-\mathrm{C}$ 键最典型的反应, 其操 作简单、条件温和、副反应少、产物纯净，对现代工业 生产极其有利. 因此, 硅氢加成反应在有机硅化学中占 有重要地位 ${ }^{[1 \sim 3]}$.

早期硅氢加成所用的催化剂以 $\mathrm{Rh}^{[4 \sim 6]} 、 \mathrm{Pt}^{[7]} 、 \mathrm{Ir}^{[8,9]}$ 、 $\mathrm{Ru}^{[10,11]}$ 等贵金属为主,如: Karstedt ${ }^{[12]}$ 催化剂、Speier ${ }^{[13]}$ 催化剂等. 这些贵金属催化剂往往具有较高的催化活 性，但存在价格昂贵，操作困难及配体复杂等缺点. 20 世纪 90 年代以来, 随着人们对各种非贵金属如 $\mathrm{Ti}^{[14]}$ 、 $\mathrm{Zn}^{[15 \sim 17]} 、 \mathrm{Sn}^{[18]} 、 \mathrm{Cu}^{[19]}$ 等催化剂体系的不断探索和深入 研究, 发现上述非贵金属催化剂在特定的条件下对烯、 酮和亚胺等双键的硅氢加成反应具有高活性与选择性. 特别是新颖的价格低廉的铁催化体系的发展, 大大拓宽 了此类非贵金属催化剂的应用前景.

非贵金属铁是参与各种生物系统必不可少的关键 元素, 相对无毒, 其丰富度在地壳所含的所有金属元素 中排在第二位, 且各种铁盐及配合物便宜易得, 极具潜
在的应用价值. 自从 Nishiyama 和 Furuta ${ }^{[20]}$ 报道了铁催 化酮的硅氢加成反应以来, 铁催化剂吸引了越来越多的 关注. 本文介绍了近些年来铁催化碳-碳双键与碳-杂原 子双键硅氢加成反应的研究进展.

\section{1 铁催化 $C=C$ 双键的硅氢加成反应}

1962 年, Nesmeyanov 课题组 ${ }^{[21]}$ 首先报道了有关羰 基铁以及铁配合物催化烯烃的硅氢加成反应. 研究发 现: $\mathrm{Fe}(\mathrm{CO})_{5}$ 催化丙烯的硅氢加成反应, 得到的是硅氢加 成和脱氢硅烷化的混合物. 催化乙烯的硅氢加成反应, 三乙基硅烷为 3 equiv.时, 产物均为硅氢加成产物; 三 乙基硅烷为 0.2 equiv. 时, 则得到脱氢硅烷化产物. 通过 改变烯烃与含氢硅烷的物质的量比, 硅氢加成和脱氢硅 烷化的产率也随之改变(Scheme 1). 含氢硅烷过量有利 于硅氢加成, 烯烃过量则有利于脱氢硅烷化. 但令人遗 憾的是, 这一现象仍然无法做出合理的解释.

随后，Murai 课题组 ${ }^{[2]}$ 报道芳基乙烯与三乙基硅烷

\footnotetext{
*E-mail: jjpeng@hznu.edu.cn; xiaowj2008@126.com

Received June 12, 2014; revised July 15, 2014; published online July 17, 2014.

Project supported by the National Natural Science Foundation of China (No. 21303035) and the Natural Science Foundation of Zhejiang Province (No. LY14B030007).

国家自然科学基金(No. 21303035)、浙江省自然科学基金(No. LY14B030007)资助项目.
} 


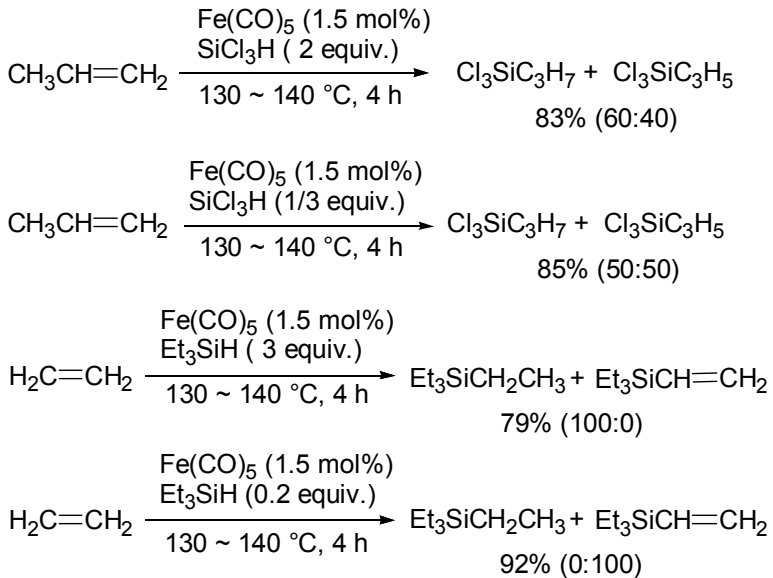

\section{Scheme 1}

在 $\mathrm{Fe}_{3}(\mathrm{CO})_{12}$ 催化下, 以苯为溶剂, $60{ }^{\circ} \mathrm{C}$ 下, 以 $66 \%$ $89 \%$ 的收率得到单一脱氢硅烷化产物(Eq. 1).

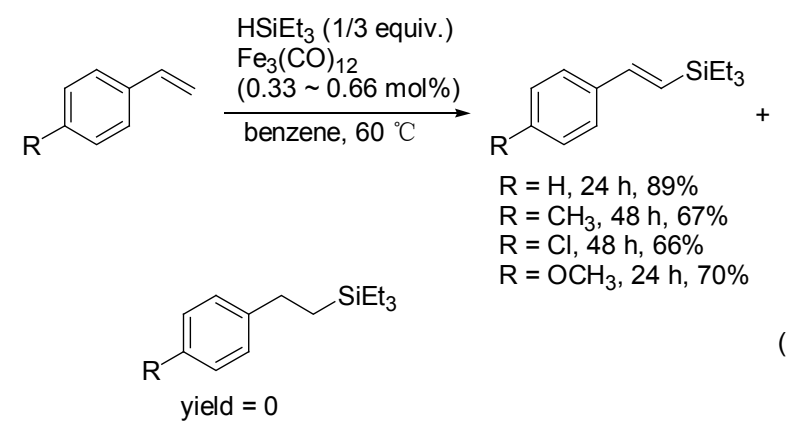

Chirik 等 ${ }^{[23]}$ 合成了一种吡啶亚胺类铁配合物催化剂 $1 \mathrm{a}$ (Eq. 2). $22{ }^{\circ} \mathrm{C}$ 下, 戊烷为溶剂, 用 $0.3 \mathrm{~mol} \% \mathbf{1 a}$ 催化 1-庚烯与苯基硅烷或二苯基硅烷反应, 产物均遵守反马 氏加成规则; 当催化其他末端烯烃和环内烯烃时, 反应 所需的时间更短. 这一催化体系选择性高, 适用范围广, 对铁催化硅氢加成反应的研究有重要的启示.

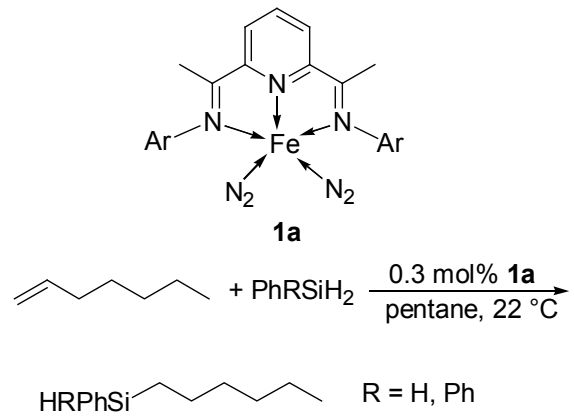

2012 年, Tondreau 等 ${ }^{[24]}$ 合成了一系列新型吡啶亚胺 类的铁配合物 $\mathbf{2} \mathbf{a} \sim \mathbf{2} \mathbf{c}(\mathrm{Eq} .3$ ), 并应用于硅氢加成反应. $23{ }^{\circ} \mathrm{C}$ 下, 以 $\left(\mathrm{Me}_{3} \mathrm{SiO}\right)_{2} \mathrm{MeSiH}$ 为氢源, 催化剂 $\mathbf{2} \mathbf{a} \sim \mathbf{2} \mathbf{c}$ 用 量分别为 $0.05 、 0.03 、 0.004 \mathrm{~mol} \%$ 时, 1-辛烯转化率达 $98 \%$ 以上. 以 $(\mathrm{EtO})_{3} \mathrm{SiH}$ 为氢源, $0.04 \mathrm{~mol} \% \mathbf{2 a}$ 为催化剂, 1-辛 烯转化率达 $96 \%$; 以 $0.02 \mathrm{~mol} \% 2 \mathrm{~b}$ 或 $0.007 \mathrm{~mol} \% 2 \mathrm{c}$ 为
催化剂, 1-辛烯转化率达 $97 \%$. 以 $\mathrm{Et}_{3} \mathrm{SiH}$ 为氢源时, 0.02 $\mathrm{mol} \%$ 2c 的催化活性最高, 1-辛烯转化率达 98\%以上, $2 \mathrm{a}$ 与 $2 \mathrm{~b}$ 则基本没有活性. 该体系催化剂用量少、活性高, 具有很大的应用潜力,为功能性有机硅化合物的制备提 供了一种独特的且比较经济的方法. 目前烯烃硅氢加成 反应所用的催化剂仍以贵金属为主 ${ }^{[25 ~ 27]}$. 鉴于铁催化 剂能达到高活性、高选选择性的效果 ${ }^{[24]}$, 其必将成为重 要的发展方向.

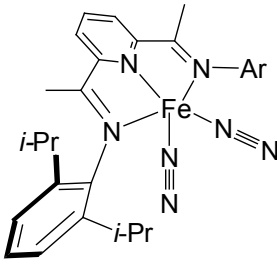

(iPrPDI) $\mathrm{Fe}\left(\mathrm{N}_{2}\right)_{2}$

2a

\section{2 铁催化 $\mathrm{C}=\mathrm{O}$ 双键的硅氢加成反应}

\section{1 铁催化醛酮的硅氢加成反应}

Brunner 和 Fish 等 ${ }^{[28,29]}$ 最先报道了 $[\mathrm{CpFe}(\mathrm{CO}) \mathrm{XL}]$ (其中 $\mathrm{X}=\mathrm{COMe}$ 或 $\mathrm{Me}, \mathrm{L}=(S)-(+)-\mathrm{Ph}_{2} \mathrm{P}[\mathrm{N}(\mathrm{Me}) \mathrm{CH}-$ $(\mathrm{Me}) \mathrm{Ph}])$, 络合物催化苯乙酮的硅氢加成反应. 随后, Beller 课题组 ${ }^{[30]}$ 对不同的铁一膦催化体系催化潜手性酮 的不对称硅氢加成反应进行了研究. 研究了配体的组成 和立体结构(图 1)对催化活性和立体选择性的影响：以 $(\mathrm{EtO})_{2} \mathrm{MeSiH}$ 为氢源, 四氢呋喃为溶剂, $\mathrm{Fe}(\mathrm{OAc})_{2}$ 分别 与配体 $3 \mathbf{a}$ 和 $\mathbf{3 b}$ 配位催化苯乙酮的硅氢加成反应, 苯乙 酮转化率达 $97 \%$ 和 $99 \%$, 但 ee 值为零; $\mathrm{Fe}(\mathrm{OAc})_{2}$ 与配体 3c 配位作催化剂时, 苯乙酮转化率达 $99 \%$, ee 值为 $25 \%$, 这一结果揭示了含磷配体的碱性越强越能够增加产物 的光学选择性. 而 $\mathrm{Fe}(\mathrm{OAc})_{2}$ 与配体 $\mathbf{3 d}$ 和配体 $\mathbf{3 f} \sim 3 \mathbf{3}$ 配 位时的苯乙酮转化率最高达 $99 \%$, ee 值最高达 $77 \%$, 这 说明配体的空间位阻越大越有利于提高其对映选择性.

2010 年, Hosokawa 课题组 ${ }^{[31]}$ 用 $\mathrm{Fe}_{2}(\mathrm{CO})_{9}$ 合成了两 种新型的铁配合物 $\mathbf{4 a} \sim \mathbf{4 b}(\mathrm{Eq} .4) .50{ }^{\circ} \mathrm{C}$ 下, 以 1.5 equiv. 的 $\mathrm{HSi}(\mathrm{OEt})_{2} \mathrm{Me}$ 为氢源，甲苯为溶剂, $2 \mathrm{~mol} \%$ 铁配 合物 $4 \mathrm{~b}$ 催化苯乙酩的硅氢加成反应, $24 \mathrm{~h}$ 后, 苯乙酩转 化率达 $40 \%$, 光学选择性达 $48 \% ; 80{ }^{\circ} \mathrm{C}$ 下, 甲苯为溶剂, 加入 $2 \mathrm{~mol} \%$ 的 $\mathrm{Na}(\mathrm{acac})$ 为添加剂, 反应 $1 \mathrm{~h}$ 后, 苯乙酮 转化率达 $99 \%$, 光学选择性达 $49 \% ; 50{ }^{\circ} \mathrm{C}$ 下, 正已烷为 


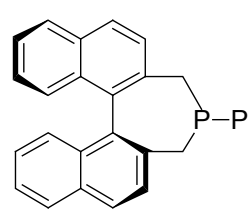

3a

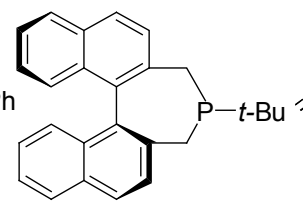

$3 b$

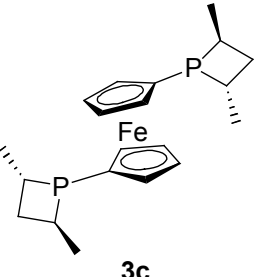

$3 c$

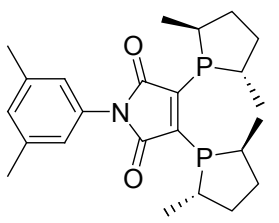

$3 f$ 3d<smiles>C[C@@H]1C2OC(C)(C)OC2C2OC(C)(C)OC2[C@@H]1C</smiles><smiles>C[C@@H]1CC[C@@H](C)P1c1c(P2[C@H](C)CC[C@H]2C)c(=O)n(C)n(C)c1=O</smiles>

$3 \mathbf{i}$

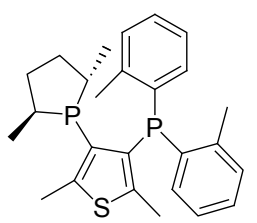

3j

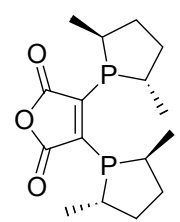

3h

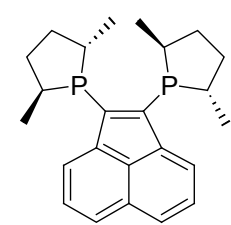

3k

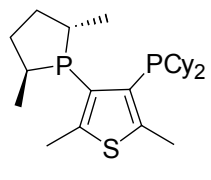

31

图 1 手型膦配体

Figure 1 Chiral phosphine ligands

溶剂, $\mathrm{Na}$ (acac)为添加剂, 反应 $24 \mathrm{~h}$ 后, 苯乙酮转化率达 $99 \%$, 光学选择性达 $66 \%$. 该反应说明添加剂能够明显 增加底物的反应速率和立体选择性.
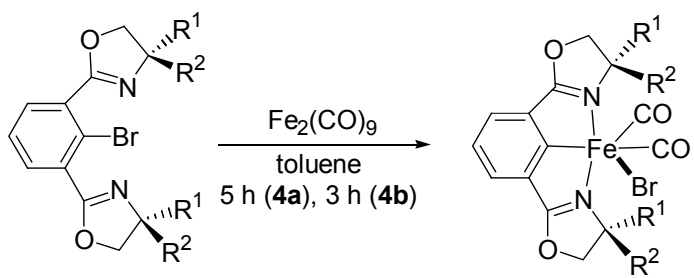

4a: $R^{1}=R^{2}=\operatorname{Me}(52 \%)$

4b: $\mathrm{R}^{1}=i-\operatorname{Pr}, \mathrm{R}^{2}=\mathrm{H}(69 \%)$

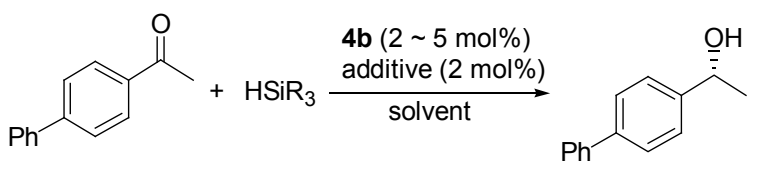

Scheme 2

Gade 课题组 ${ }^{[32]}$ 用 $N$-杂环化合物 $\mathbf{5 a} \sim \mathbf{5 c}$ (图 2) 与醋酸 亚铁配位得催化剂, 在 $65{ }^{\circ} \mathrm{C}$ 下, 以 $(\mathrm{EtO})_{2} \mathrm{MeSiH}$ 为氢 源, 四氢呋喃为溶剂, 催化䒺乙酮与苯乙酮的硅氢加成 反应. 研究发现: 配体 $5 \mathrm{c}$ 的催化活性最高, 其中菜乙酩

转化率为 $87 \%$, ee 值达 $71 \%$; 苯乙酮转化率为 $92 \%, e e$ 值达 $85 \%$. 该结果也说明配体的空间位阻越大越有利于 提高光学选择性.<smiles>CC(C)(C)C1COC(c2ccccc2Nc2ccccc2C2=N[C@@H](C(C)(C)C)CO2)=N1</smiles>

$5 a$<smiles>C/C(=N\OC1CCCCC1)c1cccc(/C(C)=N/C2CCCCC2)c1</smiles>

$5 b$

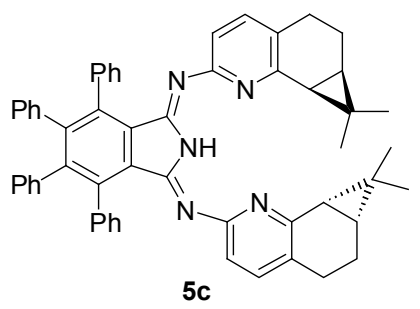

图 $25 \mathrm{a} \sim 5 \mathrm{c}$ 的结构

Figure 2 Structures of $\mathbf{5 a} \sim 5 \mathbf{c}$

Nishiyama 等 ${ }^{[33]}$ 用手性噁唑啉类配体 $\mathbf{6 a} \sim \mathbf{6 c}$ 配位醋 酸亚铁, 催化联苯乙酮的硅氢加成反应(Eq. 4). 结果发 现：使用 $6 \mathbf{a}$ 作配体，苯乙酮转化率达 93\%, ee 值达 37\%; 使用 $\mathbf{6 b}$ 作配体，苯乙酮转化率达 $82 \%$, ee 值达 $57 \%$; 使 用 $6 c$ 作配体，苯乙酩转化率达 $75 \%$, ee 值达 $79 \%$ 。

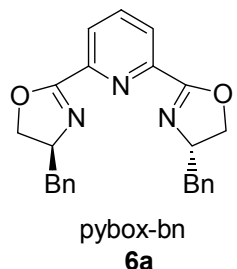

$6 a$
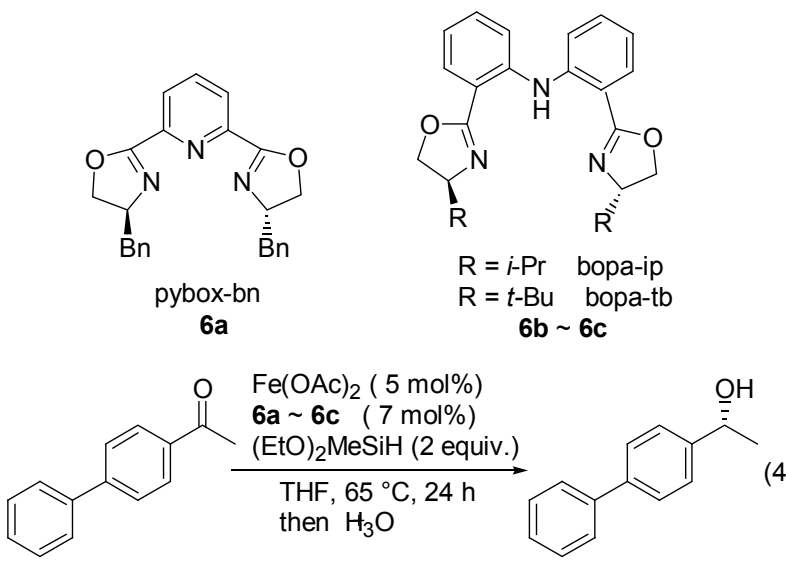

2013 年, Demir 等 ${ }^{[34]}$ 合成了一系列新的含 $\mathrm{N}$ 杂环铁 卡宾配合物 $7 \mathbf{a} \sim 7 \mathbf{f}$ 并应用于不饱和羰基化合物的硅氢 加成反应(Eq. 5) ${ }^{[35]}$. 以 $1 \mathrm{~mol} \%$ 铁卡宾配合物为催化剂, $100{ }^{\circ} \mathrm{C}$ 下催化苯基硅烷与苯甲醛硅氢加成反应， $1 \mathrm{~h}$ 后， 7a 催化活性最高, 苯甲醛转化率达 94\%, 7c 7d 的催化 活性低一些，苯甲醛转化率为 $80 \%$; 而相同条件下催化 2,5-二甲氧基苯甲醛的硅氢加成反应, $5 \mathrm{~min}$ 后, $7 \mathbf{b}$ 的催 化活性最高, 2,5-二甲氧基苯甲醛转化率达 $98 \%, 7 \mathbf{a}$ 的催 化效果较低，2,5-二甲氧基苯甲醛转化率为 $8 \%$ 。该体系 催化反应时间短，催化活性高，大大拓展了铁卡宾配合 
物在硅氢加成反应中的应用发展.

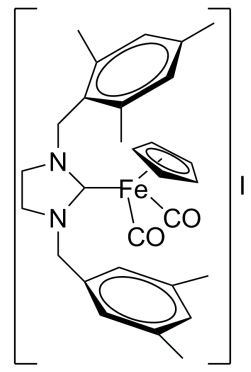

$7 a$

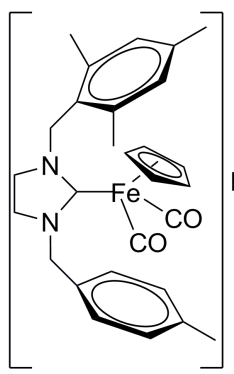

$7 d$

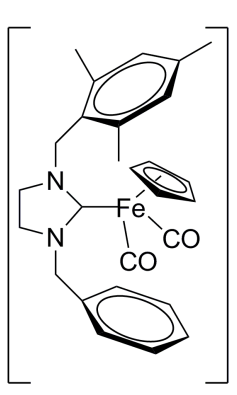

$7 b$

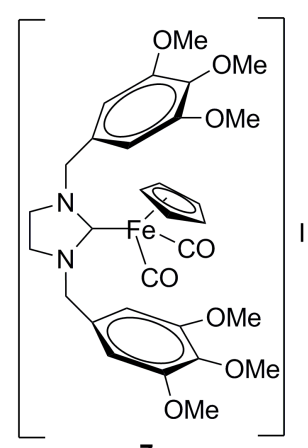

7c

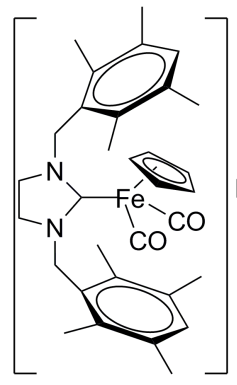

$7 e$

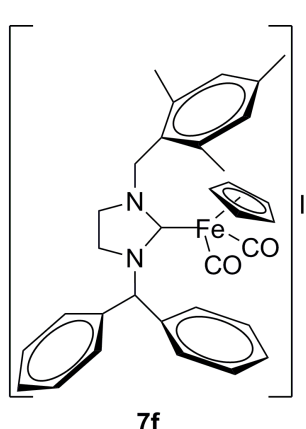

$7 f$
2) $\mathrm{NaOH/MeOH, \text {r.t. }}$

\section{2 铁催化烯酮的硅氢加成反应}

铁催化共轭 $\alpha, \beta$-不饱和羰基化合物在有机化学研究 中是一个较为活跃的领域. 产物中以醇为主. 2008 年, Furuta 和 Nishiyama 等 ${ }^{\left[{ }^{[6]}\right]}$ 报道了用铁配合物催化 $\alpha, \beta$-不 饱和芳香酮的硅氢加成(Eq. 6), 结果表明: 当 $\mathrm{R}$ 为甲基 时，仲醇的产率最高达 $93 \%$; 当 $\mathrm{R}$ 为苯基时，仲醇的产 率最高达 $57 \%$. 该催化体系说明了: 反应底物碳氧双键 上所接基团的给电子性越强, 发生 1,2-亲核加成反应的 产率越小。

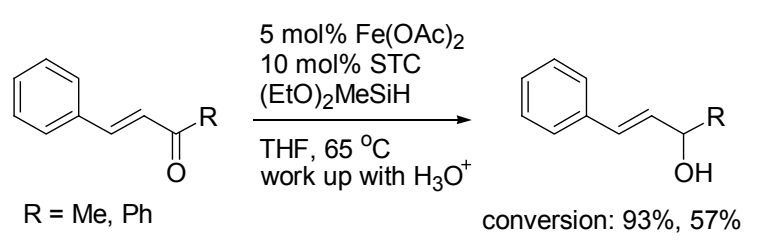

Chirik 课题组 ${ }^{[37]}$ 合成了一种新型铁配合物 8a, 并用 到烯酮硅氢加成反应中(Scheme 3). 研究发现, $0.1 \mathrm{~mol} \%$ 的铁配合物为催化剂, $23{ }^{\circ} \mathrm{C}$ 下反应 $12 \mathrm{~h}, \alpha, \beta$-不饱和环 状烯酮的硅氢加成产物均为仲醇, 而 $\alpha, \beta$-不饱和芳香酮 的硅氢加成产物则是仲醇与酮的混合物. 当底物的共轭 效应越强, 发生 1,4-加成的趋向越高.

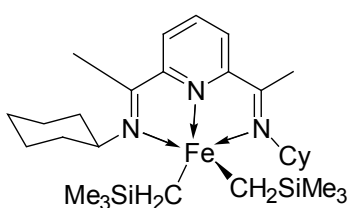

$8 a$
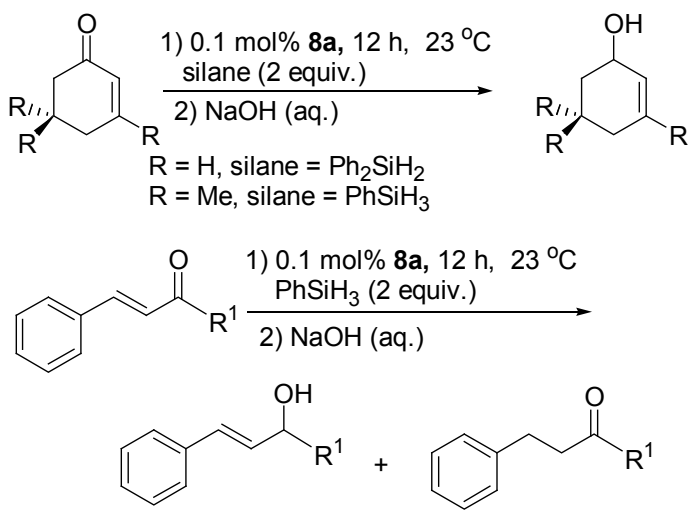

$$
\begin{array}{lll}
\mathrm{R}^{1}=\mathrm{Ph} & 7 & 1 \\
\mathrm{R}^{1}=\mathrm{Me} & 29 & 1
\end{array}
$$

\section{Scheme 3}

\section{3 铁催化酯的硅氢加成反应}

酯是羰基衍生物中的一种重要化合物，通过羰基的 催化氢化反应可以得到醇、醚等重要的化合物. 因此, 研究带酯类化合物的硅氢加成反应具有重要的理论价 值和现实意义. Sortais 等 ${ }^{[38]}$ 合成了一种新型的铁卡宾配 合物 9a (Eq. 7). $100{ }^{\circ} \mathrm{C}$ 下, $5 \mathrm{~mol} \%$ 的铁配合物 9a 催化苯 乙酸甲酯与苯基硅烷反应， $24 \mathrm{~h}$ 后，酯转化率达 $49 \%$, 其中甲基苯乙基醚与苯乙醇的比值为 $2: 3^{[39,40]}$. 产物的 混合物组成可能是由催化剂的结构特点决定.

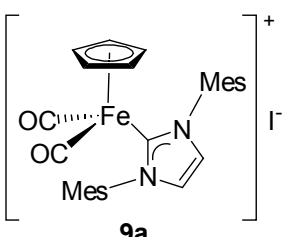

$9 a$

$$
\begin{aligned}
& \mathrm{Ph}_{\mathrm{OMe}} \frac{\begin{array}{l}
\text { 1) } \mathrm{PhSiH}_{3} \text { (4 equiv.), 9a }(5 \mathrm{~mol} \%) \\
\text { 2) } \mathrm{HCl} / \mathrm{THF}
\end{array}}{\text { neat, } 100^{\circ} \mathrm{C}, 24 \mathrm{~h}, \mathrm{hv}} \\
& \mathrm{Ph} \sim \mathrm{OMe}^{+}{ }^{\mathrm{Ph}} \curvearrowright \mathrm{OH} \\
& 49 \% \text { conversion } \\
& V(\text { ether }): V(\text { alcohol })=2: 3
\end{aligned}
$$

2013 年, Sortais 和 Darcel 等 ${ }^{[41 ~ 43]}$ 共同报道了另外一 种零价铁的卡宾配合物 10a $~ 10 \mathrm{c}(\mathrm{Eq} .8)$. 在 $350 \mathrm{~nm}$ 紫 外光的照射下, $0.5 \mathrm{~mol} \%$ 铁配合物 $\mathbf{1 0 a}$ 催化苯乙酸甲酯 与 1.1 equiv.的二乙基硅烷反应 $3 \mathrm{~h}$, 酯转化率可达 $91 \%$, 苯乙醛与苯乙醇的比值达 $88: 3$. 当催化剂 $10 \mathrm{a}$ 的用量 提高到 $1 \mathrm{~mol} \%$, 反应 $3 \mathrm{~h}$, 酯转化率达 $96 \%$, 苯乙醛与 苯乙醇的比值达 $95 ： 1$. 当 $1 \mathrm{~mol} \%$ 铁配合物 10c 催化苯 
乙酸甲酯与 1.1 equiv. 的二乙基硅烷反应 $1 \mathrm{~h}$, 酯转化率 为 $54 \%$, 苯乙醛与苯乙醇的比值为 $53: 1$. 值得注意的 是, 当催化剂 $10 \mathrm{a}$ 的用量达 $2.5 \mathrm{~mol} \%$ 时, 产物均为苯乙 醛. 该催化体系反应条件温和、催化活性高且选择性好, 极具应用潜力.<smiles>[R]N1C=CN([R])C1[P+](C)([O-])OC(=O)O</smiles>

10a $R=$ Mes $10 \mathrm{~b}=2,6-i-\mathrm{Pr}_{2} \mathrm{C}_{6} \mathrm{H}_{3}$

1) $\mathrm{Et}_{2} \mathrm{SiH}_{2}$ (1.1 equiv.)

$10 \mathrm{a} \sim 10 \mathrm{c}(0.5 \sim 2.5 \mathrm{~mol} \%)$

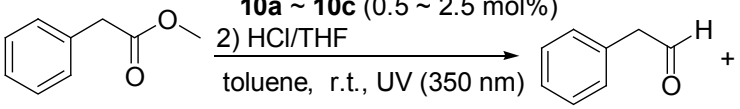<smiles>OCCc1ccccc1</smiles>

随后他们根据实验数据和模型推理提出了铁卡宾 $10 \mathrm{a}$ 催化酯的反应机理(图 3): 首先 10a 分解形成缺电子 的二价铁羰基化合物 $\mathbf{a}^{[44,45]}$, 然后与 $\mathrm{Et}_{2} \mathrm{SiH}_{2}$ 配位形成一 个新的二价铁配合物 $\mathbf{b}$, 二价铁配合物 $\mathbf{b}$ 的羰基与酯经 过还原消除反应形成含 $\mathrm{Fe}-\mathrm{Si}(\mathbf{c})^{[46 \sim 48]}$ 或者 $\mathrm{Fe}-\mathrm{H}$ (d) ${ }^{[49 \sim 51]}$ 的零价铁配合物, 释放出稳定的硅醚, 同时 $\mathrm{Fe}$ 的络合物与下一分子酯形成中间体 $\mathbf{a}$, 从而进入下一轮 催化.

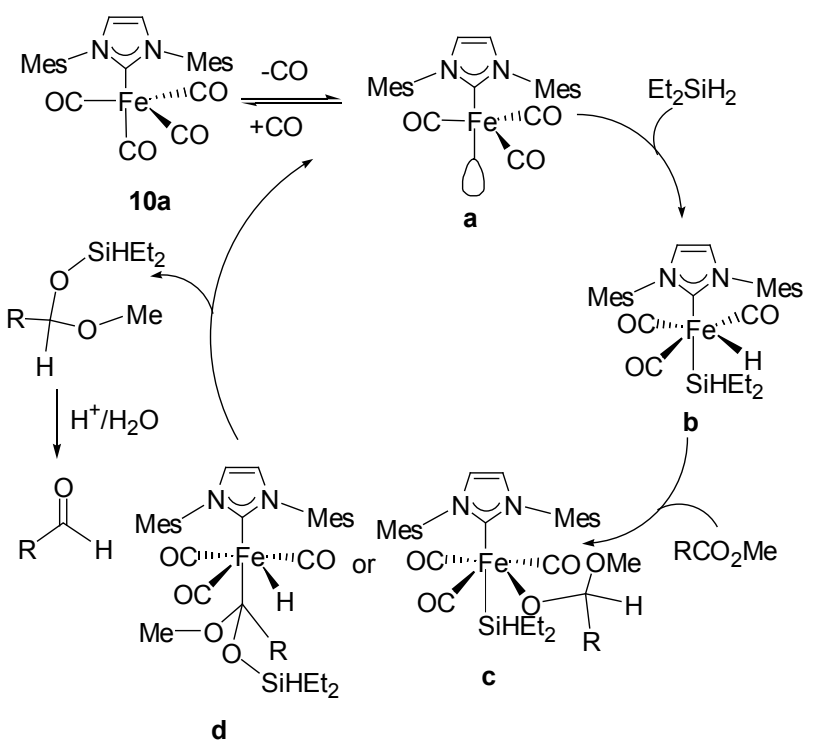

图 3 铁配合物 10a 催化酯的硅氢加成反应机理

Figure 3 Mechanism of hydrosilylation of ester catalyzed with iron complex 10a

\section{4 铁催化酰胺的硅氢加成反应}

随着研究铁催化不饱和键硅氢加成反应的不断发 展, 酰胺的硅氢加成越来越引起人们的注意. Beller 和 Nagashima 等 ${ }^{[52]}$ 共同报道了以十二羰基三铁为催化剂,
PMHS 和 TMDS 为氢源, $100{ }^{\circ} \mathrm{C}$ 下，回流 $24 \mathrm{~h}$ (或在室温 的条件下, $400 \mathrm{~W}$ 永灯照射 $9 \mathrm{~h}$ ), 催化不同二、三级酰胺 的硅氢加成, 酰胺转化率达 $25 \% \sim 98 \%$. Sortais 等 ${ }^{[53]}$ 合 成的铁卡宾配合物 9a 也用于催化不同二、三级酰胺的 硅氢加成(Scheme 4). $100{ }^{\circ} \mathrm{C}$ 下, 光辐射 $24 \mathrm{~h}, 5 \mathrm{~mol} \%$ 的 铁卡宾催化 2 equiv.的苯基硅烷与酰胺反应, $24 \mathrm{~h}$ 后, 酰 胺转化率可达 77\% 98\%, 表明这种催化剂几乎适用于 所有的芳香族和脂肪族酰胺. 相同的条件下, 当 $9 \mathrm{a}$ 催化 一级酰胺时, 产物几乎全为含氧基的衍生物.

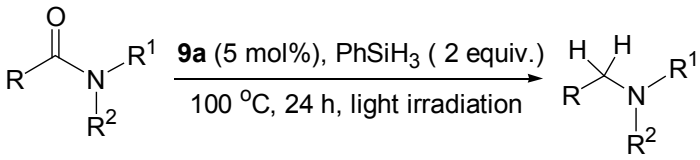

$$
\begin{aligned}
& \mathrm{R}_{\mathrm{N}_{\mathrm{H}}^{-}}^{\stackrel{\mathrm{O}}{\mathrm{H}} \underset{100^{\circ} \mathrm{C}, 24 \mathrm{~h} \text {, light irradiation }}{\mathrm{9a}(5 \mathrm{~mol} \%), \mathrm{PhSiH}_{3} \text { ( } 2 \text { equiv.) }}} \mathrm{R}-\mathrm{C}=\mathrm{N}
\end{aligned}
$$

\section{Scheme 4}

Adolfsson 研究小组 ${ }^{[54]}$ 制备了一种咪唑类杂环 11a, 将其作为醋酸亚铁的配体, 催化酰胺的硅氢加成反应 (Scheme 5). 在 $65{ }^{\circ} \mathrm{C}$ 下, 四氢呋喃作溶剂, 芳香族酰胺 的产率达 $78 \% \sim 92 \%$. 随后，他们依据是否添加 $\mathrm{LiCl}$, 出现副产物醛和醇的情况提出了三级酰胺硅氢加成的 机理: 首先, 三级酰胺与含氢硅烷发生加成反应, 形成 四面中间体 $\mathbf{a}$ ，中间体经过消除反应生成亚胺离子 $\mathbf{b}$, 亚 胺离子与另外一个含氢硅烷反应生成产物 c. 而形成醇 的途径则有两条(Scheme 6): 一条是通过中间体 $\mathbf{a}$ 形成 带有正电的氧鎓离子 $\mathbf{d}$; 另一条是亚胺离子 $\mathbf{b}$ 通过消除 仲氨形成醛 $\mathbf{e}$, 然后形成醇 $\mathbf{f}$. 当有 $1 \mathrm{~mol} \%$ 的 $\mathrm{LiCl}$ 加入 时, 四面中间体能快速形成亚胺离子, 从而增加胺的转 化率. 但遗憾的是, 这一反应机理仅限于三级酰胺的硅 氢加成反应.
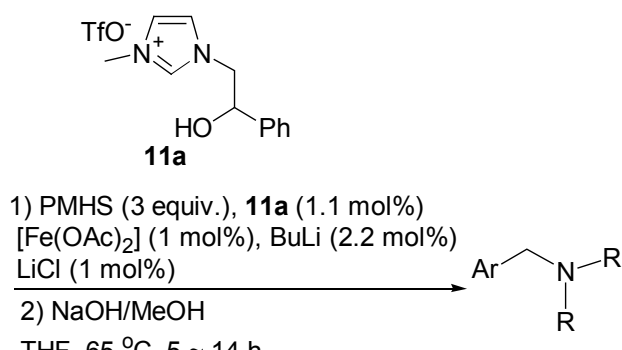

$\mathrm{THF}, 65^{\circ} \mathrm{C}, 5 \sim 14 \mathrm{~h}$

\section{Scheme 5}

\section{3 铁催化其他杂原子双键的硅氢加成反应}

\section{1 铁催化 $\mathrm{C}=\mathrm{N}$ 双键的硅氢加成反应}

由于胺在天然产物中广泛存在及生产生活中的广 泛应用,因此亚胺的硅氢化具有重要的意义, 但是用金 


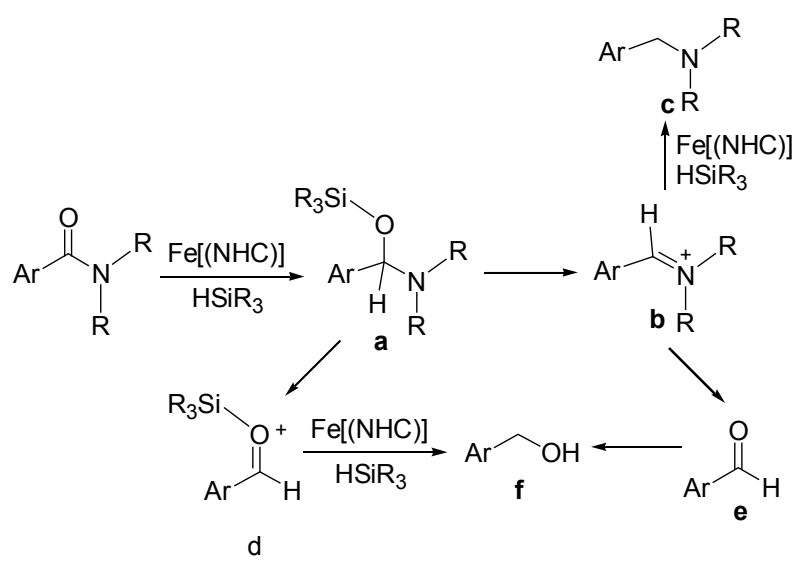

Scheme 6

属铁催化亚胺类化合物的硅氢加成至今仍处于初级阶 段. 2010 年, Beller 课题组 ${ }^{[55]}$ 第一个报道了关于铁配合物 催化 $N$-(二苯基膦基)-酮亚胺的硅氢化反应, 这给研究 者们用铁催化 $\mathrm{C}=\mathrm{N}$ 不饱和双键硅氢加成反应带来了新 的启发. 2012 年, Darcel 课题组 ${ }^{[56]}$ 合成了一种新型的铁 配合物 12a，并第一次运用到亚胺的硅氢加成反应中 (Scheme 7). 在 $30{ }^{\circ} \mathrm{C}$ 下, $2 \mathrm{~mol} \%$ 的铁配合物催化醛亚胺 与 2 equiv. 苯基硅烷硅氢加成反应, $30 \mathrm{~h}$ 后, 醛亚胺类化 合物转化率达 $10 \% \sim 95 \%$; 而在 $100{ }^{\circ} \mathrm{C}$ 下, $5 \mathrm{~mol} \%$ 的铁 配合物催化酮亚胺与 2 equiv. 苯基硅烷硅氢加成反应, $24 \mathrm{~h}$ 后，酮亚胺类化合物转化率达 57\% 95\%. 与催化 醛亚胺相比, 催化酮亚胺反应条件更苛刻, 但胺的产率 更好.
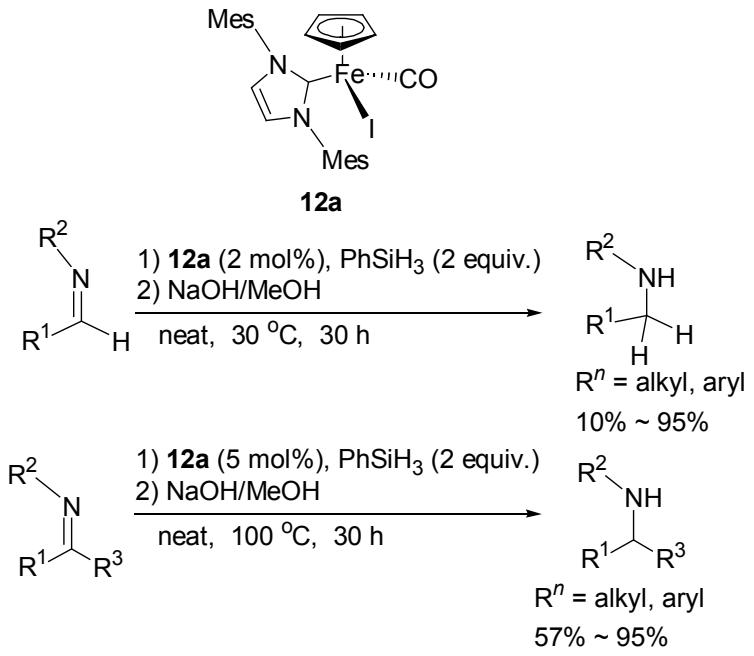

\section{Scheme 7}

\section{2 铁催化 $\mathrm{S}=\mathrm{O}$ 双键的硅氢加成反应}

亚砜的化学性质比较稳定, 一般很难发生硅氢化反 应，目前关于亚砜的硅氢加成反应报道很少. 2011 年, Enthaler 课题组 ${ }^{[57]}$ 第一次报道了有关铁催化亚砜的硅氢
加成反应. 在 $100{ }^{\circ} \mathrm{C}$ 下, 甲苯作溶剂, 以 5 equiv. 的 PMHS 或 1 equiv. 的 $\mathrm{PhSiH}_{3}$ 为氢源, $5 \sim 10 \mathrm{~mol} \%$ 的 $\mathrm{Fe}_{2}(\mathrm{CO})_{9}$ 为催化剂, 反应 $24 \mathrm{~h}$ 后得硫醚. 最近, Royo 课 题组 ${ }^{[58}$ 报道了一种用咪唑盐与 $\mathrm{Fe}_{3}(\mathrm{CO})_{12}$ 合成的新型铁 配合物 13a (Scheme 8). $100{ }^{\circ} \mathrm{C}$ 下，用 $1 \mathrm{~mol} \%$ 的 13a 催化 不同亚砜与 2 equiv.的苯基硅烷反应， $6 \mathrm{~h}$ 后，二甲亚砜 的转化率达 66\%, 苯甲亚砜的转化率达 $99 \%$. 经过大量 的实验他们得出，当 $\mathrm{R}^{n}$ 为氯或者乙烯基时，光学选择性 减少; 当 $\mathrm{R}^{n}$ 为乙酸甲酯时，则几乎不发生反应. 经过一 系列催化不同底物的实验，他们证明该反应遵循自由基 机理进行.
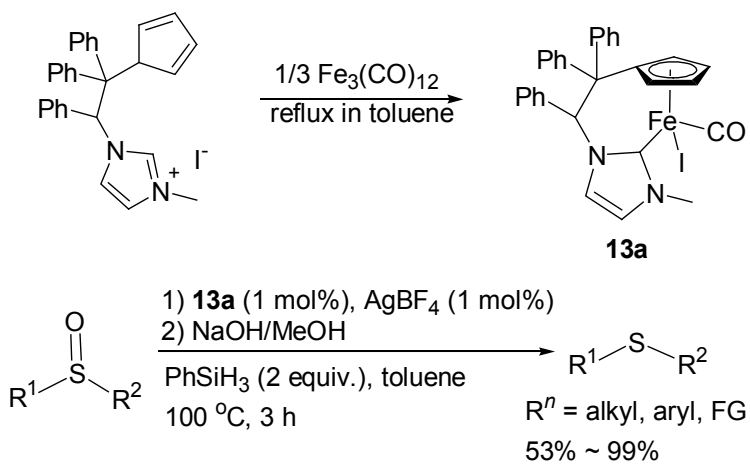

Scheme 8

\section{4 结论与展望}

铁配合物目前已广泛用于双键的硅氢加成反应. 但 是，在碳一碳双键硅氢加成方面，铁催化的例子依然很 少，所报道的配体也只有少数几种; 而贵金属催化文献 报道却较多, 如 Karstedt 催化剂、Speier 催化剂甚至已 经应用到工业生产中. 在碳一氧等杂原子双键方面，大 多数反应中铁催化硅氢加成反应的副产物较多, 反应活 性与选择性不高，且机理不明确; 而贵金属如 $\mathrm{Pt}$ 等具有 催化反应产物均一, 反应活性与立体选择高, 机理研究 较透彻等优点.

铁催化不饱和双键硅氢加成的应用在催化研究中 占有非常重要的地位,尽管已经取得了不少成绩，但这 一领域仍然有许多尚未解决的问题,如催化剂在空气中 的稳定性、操作复杂、反应条件苛刻等, 因此合成新颖 的、简单的、可操作性强的、高效性(高活性、高选择 性、高回收性)的配体仍然是今后研究的重要内容.

\section{References}

[1] Xing, S. M.; Wang, Y. L. Organic Silicon Synthesis Technology and Product Application, Chemistry Industry Press, Beijing, 2000, p. 138 (in Chinese).

(幸松民，王一璐，有机硅合成工艺及产品应用，化学工业出版 社, 北京, 2000, p. 138.)

[2] Junge, K.; Schröder, K.; Beller, M. J. Chem. Commun. 2011, 47, 
4849.

[3] Rossiter, K. J. J. Chem. Rev. 1996, 96, 3201.

[4] Dong, H.; Berke, H. Adv. Synth. Catal. 2009, 351, 1783.

[5] Ojima, I.; Kogure, T.; Nagai, Y. Tetrahedron Lett. 1974, 15, 1889.

[6] Brunner, H.; Becker, R.; Riepl, G. Organometallics 1984, 3, 1354.

[7] Igarashi, M.; Fuchikami, T. Tetrahedron Lett. 2001, 42, 1945.

[8] Nishibayashi, Y.; Segawa, K.; Ohe, K.; Uemura, S. Organometallics 1995, 14, 5486.

[9] Chen, T.; Liu, X. G.; Shi, M. Tetrahedron 2007, 63, 4874.

[10] Enders, D.; Gielen, H.; Breuer, K. Tetrahedron: Asymmetry 1997, $8,3571$.

[11] Zhu, G.; Terry, M.; Zhang, X. J. Organomet. Chem. 1997, 547, 97.

[12] Karstedt, B. D. FR 1548775, 1968 [Chem. Abstr. 1969, 71, 91641]

[13] Speier, J. L.; Webster, J. A.; Barnes, G. H. J. Am. Chem. Soc. 1957, 79,574 .

[14] Yun, J.; Buchwald, S. L. J. Am. Chem. Soc. 1999, 121, 5640.

[15] Bette, V.; Mortreux, A.; Savoia, D.; Carpentierc, J. F. Adv. Synth. Catal. 2005, 347, 289.

[16] Gajewy, J.; Kwit, M.; Gawroński, J. Adv. Synth. Catal. 2009, 351, 1055.

[17] Liu, S.; Peng, J. J.; Yang, H.; Li, J. Y.; Bai, Y.; Lai, G. Q. Tetrahedron 2012, 68,1371.

[18] Lawrence, N.; Bushell, S. M. Tetrahedron. Lett. 2000, 41, 4507.

[19] Diez-Gonzalez, S.; Nolan, S. P. Acc. Chem. Res. 2008, 41, 349.

[20] Nishiyama, H.; Furuta, A. Chem. Commun. 2007, 760.

[21] Nesmeyanov, A. N.; Freidlina, R. K.; Chukovskaya, E. C.; Petrova, R. G.; Belyavsky, A. B. Tetrahedron 1962, 17, 61.

[22] Kakiuchi, F.; Tanaka, Y.; Chatani, N.; Murai, S. J. Organomet. Chem. 1993, 456, 45.

[23] Bart, S. C.; Lobkovsky, E.; Chirik, P. J. J. Am. Chem. Soc. 2004, 126, 13794.

[24] Tondreau, A. M.; Atienza, C. C. H.; Weller, K. J.; Nye, S. A.; Lewis, K. M.; Delis, J. G. P.; Chirik, P. J. Science 2012. 335. 567.

[25] Chianese, A. R.; Crabtree, R. H. Organometallics 2005, 24, 4432.

[26] César, V.; Gade, L. H.; Bellemin-Laponnaz, S. Angew. Chem. 2004, 116, 1036.

[27] Tao, B.; Fu, G. C. Angew. Chem. 2002, 114, 4048.

[28] Brunner, H.; Eder, R.; Hammer, B.; Klement, U. J. Organomet. Chem. 1990, 394, 555.

[29] Brunner, H.; Fisch, K. Angew. Chem., Int. Ed. Engl. 1990, 29, 1131.

[30] Shaikh, N. S.; Enthaler, S.; Junge, K.; Beller, M. Angew. Chem., Int. Ed. 2008, 47, 2497.

[31] Hosokawa, S.; Ito, J.; Nishiyama, H. Organometallics 2010, 29, 5773.

[32] Langlotz, B. K.; Wadepohl, H.; Gade, L. H. Angew. Chem., Int. Ed. 2008, 47,4670 .

[33] Inagaki, T.; Ito, A.; Ito, J. I.; Nishiyama, H. Angew. Chem. Int. Ed. 2010, 49, 9384.

[34] Demir, S.; Gökçe, Y.; Kaloğlu, N.; Sortais, J-B.; Darcel, C.; Öz- demir, İ. Appl. Organomet. Chem. 2013. 459.

[35] Kandepi, V. V. K. M.; Cardoso, J. M. S.; Peris, E.; Royo, B. Organometallics 2010, 29, 2777.

[36] Furuta, A.; Nishiyama, H. Tetrahedron Lett. 2008, 49, 110.

[37] Tondreau, A. M.; Lobkovsky, E.; Chirik, P. J. Org. Lett. 2008, 10, 2789.

[38] Bézier, D.; Venkanna, G. T.; Misal Castro, L. C.; Zheng, J.; Roisnel, T.; Sortais, J.-B.; Darcel, C. Adv. Synth. Catal. 2012, 354, 1879.

[39] Junge, K.; Wendt, B.; Zhou, S.; Beller, M. Eur. J. Org. Chem. 2013, 2061.

[40] Das, S.; Li, Y.; Junge, K.; Beller, M. Chem. Commun. 2012, 48, 10742 .

[41] Li, H.; Misal Castro, L. C.; Zheng, J.; Roisnel, T.; Dorcet, V.; Sortais, J.-B.; Darcel, C. Angew. Chem., Int. Ed. 2013, 52, 8045.

[42] Warratz, S.; Postigo, L.; Royo, B. Organometallics 2013, 32, 893.

[43] Huttner, G.; Gartzke, W. Chem. Ber. 1972, 105, 2714.

[44] Graff, J. L.; Sanner, R. D.; Wrighton, M. Organometallics 1982, 1, 837.

[45] Liu, D. K.; Brinkley, C. G.; Wrighton, M. S. Organometallics 1984, $3,1449$.

[46] Reinhard, G.; Hirle, H.; Schubert, U. J. Organomet. Chem. 1992, $427,173$.

[47] Reinhard, G.; Hide, B.; Schubert, U.; Knorr, M.; Braunstein, P.; Mian, A.; Fischers, J. Inorg. Chem. 1993, 32, 1656.

[48] Pugh, D.; Wells, N. J.; Evans, D. J.; Danopoulos, A. A. Dalton Trans. 2009, 7189.

[49] Brinkley, C. G.; Dewan, J. C.; Wrighton, M. S. Inorg. Chim. Acta 1986, $121,119$.

[50] Langer, R.; Diskin-Posner, Y.; Leitus, G.; Shimon, L. J. W.; Ben-David, Y.; Milstein, D. Angew. Chem., Int. Ed. 2011, 50, 9948.

[51] Danopoulos, A. A.; Pugh, D.; Smith, H.; Saßmannshausen, J. Chem. Eur. J. 2009, 15, 5491.

[52] (a) Zhou, S.; Junge, K.; Addis, D.; Das, S.; Beller, M. Angew. Chem., Int. Ed. 2009, 48, 9507.

(b) Sunada, Y.; Kawakami, H.; Imaoka, T.; Motoyama, Y.; Nagashima, H. Angew. Chem., Int. Ed. 2009, 48, 9511.

(c) Tsutsumi, H.; Sunada, Y.; Nagashima, H. Chem. Commum. 2011, 47, 6581 .

[53] Zier, D. B.; Venkanna, G. T.; Sortais, J.-B.; Darcel, C. ChemCatChem 2011, 3, 1747

[54] Volkov, A.; Buitrago, E.; Adolfsson, H. Eur. J. Org. Chem. 2013, 2066.

[55] Zhou, S.; Fleischer, S.; Junge, K.; Das, S.; Addis, D.; Beller, M. Angew. Chem., Int. Ed. 2010, 49, 8121.

[56] Misal Castro, L. C.; Sortais, J.-B.; Darcel, C. Chem. Commun. 2012, $48,151$.

[57] Enthaler, S. Chem CatChem 2011, 3, 666.

[58] Cardoso, J. M. S.; Royo, B. Chem. Commun. 2012, 48, 4944. 